\title{
Association of DNA repair gene polymorphisms with response to chemotherapy and prognosis of gastric cancer
}

\author{
Z.H. Chen, L. Wang and L.P. Luo \\ The First Affiliated Hospital of Jinan University, Guangzhou, China \\ Corresponding author: L.P. Luo \\ E-mail: luoliap_ju@163.com
}

Genet. Mol. Res. 13 (3): 7484-7491 (2014)

Received July 25, 2013

Accepted January 13, 2014

Published September 12, 2014

DOI http://dx.doi.org/10.4238/2014.September.12.15

\begin{abstract}
We investigated the correlation between the response to chemotherapy in patients and excision repair cross-complimentary group 1 gene $(E R C C 1)$ and xeroderma pigmentosum complementation group $\mathrm{F}$ gene $(X P F)$ polymorphisms and the effect of these polymorphisms on the clinical outcome of gastric cancer. Samples from a total of 255 patients with newly diagnosed and histopathologically confirmed primary gastric cancer were collected in our study. The ERCC1 rs11615, ERCC1 rs2298881, XPF rs2276465, and $X P F$ rs6498486 polymorphisms were genotyped. Among the 255 patients, the median follow-up time was 29.7 months. A total of 103 patients $(40.4 \%)$ died from gastric cancer during the follow-up period. We observed that the $X P F$ rs6498486 CC genotype and the $X P F$ rs2276465 GG genotype were associated with response to chemotherapy, with odds ratios and $95 \%$ confidence intervals of 3.88 (1.23-16.07) and 2.66 (1.17-6.45), respectively. In the Cox proportional hazards model, patients carrying the ERCC1 rs11615 AA genotype and the $X P F$ rs 2276465 GG genotype showed only a 0.22 - and
\end{abstract}


0.30 -fold increased risk of death from gastric cancer. We found that the $X P F$ rs6498486 and $X P F$ rs2276465 polymorphisms are markers of response to oxaliplatin/5-fluorouracil-based chemotherapy in gastric cancer patients.

Key words: ERCC1; XPF; Gastric cancer; Response; Oxaliplatin/5-Fu-survival

\section{INTRODUCTION}

Gastric cancer is the fourth most common cancer worldwide with an estimated 988,000 new cases and 736,000 deaths each year [International Agency for Research on Cancer (IARC), 2008]. In China, gastric cancer is the second-most common cause of cancer-related deaths, killing approximately 231,193 people in 2008 [International Agency for Research on Cancer (IARC), 2008]. Although the incidence of non-cardia gastric cancer has slightly decreased in the last 2 decades, the incidence of gastric cardia cancer shows a significantly increased trend in China. Surgery is the primary modality for managing early-stage disease, but most patients develop local or distant recurrence (MacDonald, 2004). 5-Fluorouracil (5-FU) remains the main chemotherapeutic agent for treating gastric cancer, and combination chemotherapy with 5-FU has shown improved clinical outcomes (Wohrer et al., 2004); however, many patients show different prognoses even when they received the same treatment. This suggests that inter-individual variation in drug metabolizing enzymes may influence the prognosis of gastric cancer.

Recently, several studies have shown that single nucleotide polymorphisms (SNPs) influence the mechanisms of DNA repair function and play a role in removing DNA adducts. Therefore, SNPs may also affect the response to chemotherapy. Repair of DNA damage is a complex process involving a series of DNA repair pathways. In humans, more than 130 genes participate in the DNA repair process (Wood et al., 2001). Nucleotide excision repair (NER) is the most versatile DNA repair mechanism pathway and is responsible for removing a wide variety of DNA lesions, such as bulky adducts, cross-links, oxidative DNA damage, alkylating damage, and thymidine dimers (De Silva et al., 2000; Friedberg, 2001; Wood et al., 2001). The excision repair cross-complimentary group 1 gene (ERCC1) encodes a subunit of the NER complex required for the incision step of NER, which forms a heterodimer with the xeroderma pigmentosum complementation group $\mathrm{F}(\mathrm{XPF})$ endonuclease to catalyze the 5 ' incision during excision of the DNA lesion (Wang et al., 2011). Previous studies have indicated that $E R C C 1-X P F$ genetic variation is associated with prognosis of various cancers, such as ovarian cancer, head and neck cancer, and breast cancer (Carles et al., 2006; Alexander et al., 2010; Vaezi et al., 2011; DeLoia et al., 2012; Fleming et al., 2012). However, only one study conducted in the United Kingdom demonstrated the role of ERCC1 and XPF polymorphisms in response to chemotherapy and in the prognosis of gastric cancer.

There have been no studies conducted in China to examine whether ERCC1-XPF gene polymorphisms correlate with survival or response chemotherapy in cancer patients. The goal of the present study was to identify whether a correlation exists between ERCC1-XPF gene polymorphisms and the response to chemotherapy and the role of these 2 gene polymorphisms on the clinical outcome of gastric cancer. 


\section{MATERIAL AND METHODS}

\section{Patient eligibility}

A total of 255 patients with newly diagnosed and histopathologically confirmed primary gastric cancer were recruited for our study, including gastric cardia adenocarcinoma and non-gastric cardia adenocarcinoma cases. All cases were selected from patients of the Traditional Chinese Medicine of Chongqing and Chongqing Medical University between January 2007 and January 2008. Cases with secondary or recurrent tumors were excluded. Patients were ineligible if they had inadequate organ function, were pregnant, or breast-feeding. All patients provided written informed consent prior to chemotherapy. The protocol of our study was approved by The First Affiliated Hospital of Jinan University.

\section{Chemotherapy}

The 255 patients were treated with one of the following fluorouracil-based chemotherapy programs: $500 \mathrm{mg} / \mathrm{m}^{2} 5$-FU for five days and $2500 \mathrm{mg} / \mathrm{m}^{2}$ capecitabine for 2 weeks. Conjunctive chemotherapy included $25 \mathrm{mg} / \mathrm{m}^{2}$ cisplatin for 3 days, $60 \mathrm{mg} / \mathrm{m}^{2}$ oxaliplatin for 8 days, $75 \mathrm{mg} /$ $\mathrm{m}^{2}$ docetaxel for 1 day, or $60 \mathrm{mg} / \mathrm{m}^{2}$ paclitaxel for 8 days. The response to treatment was evaluated according to the World Health Organization (WHO) criteria (Miller et al., 1981). Complete remission (CR) and partial remission (PR) were considered to be responsive, and stable disease (SD) and progressive disease (PD) were considered as non-responsive. Overall survival (OS) was calculated from the date of chemotherapy to the date of death or last clinical follow-up.

\section{Genotyping}

Potential ERCC1 and XPF SNPs were identified in the National Center for Biotechnology Information (NCBI) dbSNP database according to the following criteria: minor allele frequency of selected SNPs was $\geq 10 \%$ in the Chinese population; (2) affected microRNA binding site activity; (3) not included in the published genome-wide association study. A total of 2 SNPs in ERCC1 (rs11615 and rs2298881) and 2 SNPs in XPF (rs2276465 and rs6498486) were selected. Genomic DNA was extracted from the buffy-coat fraction of the blood sample with the Qiagen Blood DNA Mini Kit (Qiagen; Hilden, Germany) according to manufacturer instructions. DNA purity and concentration were determined by spectrophotometric absorbance measurements at $260 \mathrm{~nm}$ and $280 \mathrm{~nm}$ using an ultraviolet spectrophotometer (Nano Drop Technologies, Inc.; Wilmington, DE, USA).

ERCC1 rs11615, ERCC1 rs2298881, XPF rs2276465, and XPF rs6498486 were genotyped using Taqman assays in the ABI Prism 7911HT Sequence Detection System (Applied Biosystems; Foster City, CA, USA). Primers, probes, and reaction conditions are available upon request. Genotyping was conducted by laboratory personnel who were blinded to the status of cases. For quality control, we randomly selected $10 \%$ of the samples for repeated genotyping, and the results showed $100 \%$ concordance.

\section{Statistical analysis}

All analyses were performed using the SPSS version 16.0 statistical software (SPSS; 
Chicago, IL, USA). Continuous variables are expressed as the mean \pm standard deviation (SD), whereas categorical variables are shown as frequencies and percentages. Demographic characteristics were compared between cases and controls using $\chi^{2}$ and Student $t$-tests. Survival distributions were estimated using the Kaplan-Meier method and assessed using the logrank test. The association between genotype and survival was estimated based on the hazard ratios (HRs) and their confidence intervals (CIs) from a multivariate Cox proportional hazards model. A two-sided $\mathrm{P}$ value $<0.05$ was considered to be significant.

\section{RESULTS}

There were 255 patients ( 85 females/170 males). The median age was $55.7 \pm 9.3$ years (Table 1).

\begin{tabular}{|c|c|c|}
\hline Characteristics & Cases $(\mathrm{N}=255)$ & $\%$ \\
\hline Age (means \pm SD) & $55.7 \pm 9.3$ & \\
\hline \multicolumn{3}{|l|}{ Gender } \\
\hline Female & 85 & 33.3 \\
\hline Male & 170 & 66.7 \\
\hline \multicolumn{3}{|l|}{ Smoking } \\
\hline Never & 169 & 66.3 \\
\hline Ever & 86 & 33.7 \\
\hline \multicolumn{3}{|l|}{ Drinking } \\
\hline Never & 167 & 65.5 \\
\hline Ever & 88 & 34.5 \\
\hline \multicolumn{3}{|c|}{ Family history of gastric cancer in first-relatives } \\
\hline No & 234 & 91.8 \\
\hline Yes & 21 & 8.2 \\
\hline \multicolumn{3}{|l|}{ Tumor histology } \\
\hline Intestinal & 142 & 55.7 \\
\hline Diffuse & 104 & 40.8 \\
\hline Other & 9 & 3.5 \\
\hline \multicolumn{3}{|l|}{ TNM stage } \\
\hline $\mathrm{I}-\mathrm{II}$ & 100 & 39.2 \\
\hline III-IV & 155 & 60.8 \\
\hline
\end{tabular}

All patients were followed up from the date of diagnosis until the end of December 2012. The median follow-up time was 29.7 months, and ranged from 2-60 months. During the follow-up period, 103 patients $(40.4 \%)$ died from gastric cancer.

One hundred and fifty six patients showed responsive to chemotherapy (CR or PR), with a response rate of $61.2 \%$ (Table 2). Patients were classified into responders and non-responders, and a significantly different genetic distributions of $X P F$ rs6498486 and $X P F$ rs2276465 were observed between these groups, and significantly higher $X P F$ rs6498486 CC and $X P F$ rs6498486 $\mathrm{CC}$ frequencies were observed compared to the wild-type genotype, with ORs $(95 \% \mathrm{CI})$ of 3.88 (1.23-16.07) and 2.66 (1.17-6.45), respectively. However, we observed no significant betweengroup differences in the frequencies of ERCC1 rs11615 and ERCC1 rs2298881.

Patients carrying the $X P F$ rs6498486 CC genotype showed a significantly longer median survival time (34.7 months) than those carrying the AA genotype (26.4 months). By the log-rank test, a significantly difference was found in survival times (Table 3).

In the Cox proportional hazards model, after adjusting for potential confounding 
factors, the HR $(95 \% \mathrm{CI})$ for OS in patients carrying the XPF rs6498486 CC genotype was 0.22 (0.05-0.69) using the AA genotype as the reference variable. Patients carrying the $X P F$ rs2276465 GG genotype was associate with a significantly decreased risk of death from gastric cancer when compared with those carrying the CC genotype, and the $\mathrm{HR}(95 \% \mathrm{CI})$ of overall survival was $0.30(0.12-0.69)$.

However, we observed no significant association between the ERCC1 rs11615 and $E R C C 1$ rs2298881 polymorphisms and survival time in gastric cancer patients.

Table 2. ERCC1-XPF polymorphism response to chemotherapy.

\begin{tabular}{|c|c|c|c|c|c|c|c|c|}
\hline & Total frequencies & $\%$ & $\begin{array}{c}\text { Responders } \\
\mathrm{N}=156\end{array}$ & $\%$ & $\begin{array}{c}\text { Non-responders } \\
\quad \mathrm{N}=99\end{array}$ & $\%$ & $\mathrm{P}$ & OR $(95 \% \mathrm{CI})$ \\
\hline \multicolumn{9}{|c|}{ ERCC1 rs11615 } \\
\hline GG & 111 & 43.5 & 65 & 41.6 & 46 & 46.5 & - & 1.0 (Reference) \\
\hline AG & 97 & 38.1 & 58 & 37.3 & 39 & 39.4 & 0.86 & $1.05(0.58-1.90)$ \\
\hline AA & 47 & 18.4 & 33 & 21.1 & 14 & 14.1 & 0.17 & $1.67(0.76-3.76)$ \\
\hline \multicolumn{9}{|c|}{ ERCC1 rs2298881 } \\
\hline AA & 165 & 64.7 & 98 & 62.9 & 67 & 67.5 & - & 1.0 (Reference) \\
\hline $\mathrm{AC}$ & 58 & 22.8 & 34 & 21.7 & 24 & 24.5 & 0.92 & $0.97(0.51-1.87)$ \\
\hline $\mathrm{CC}$ & 32 & 12.5 & 24 & 15.4 & 8 & 7.9 & 0.1 & $2.05(0.83-5.59)$ \\
\hline \multicolumn{9}{|c|}{ XPF rs6498486 } \\
\hline AA & 174 & 68.2 & 102 & 65.4 & 72 & 72.6 & - & 1.0 (Reference) \\
\hline $\mathrm{AC}$ & 55 & 21.6 & 32 & 20.5 & 23 & 23.3 & 0.95 & $0.98(0.51-1.91)$ \\
\hline $\mathrm{CC}$ & 26 & 10.2 & 22 & 14.1 & 4 & 4.1 & 0.01 & $3.88(1.23-16.07)$ \\
\hline \multicolumn{9}{|c|}{ XPF rs2276465 } \\
\hline $\mathrm{CC}$ & 157 & 61.5 & 87 & 55.8 & 70 & 70.5 & - & 1.0 (Reference) \\
\hline $\mathrm{CG}$ & 55 & 21.6 & 36 & 23.1 & 19 & 19.2 & 0.19 & $1.52(0.77-3.07)$ \\
\hline GG & 43 & 16.9 & 33 & 21.1 & 10 & 10.3 & 0.01 & $2.66(1.17-6.45)$ \\
\hline
\end{tabular}

Table 3. Univariate analysis of ERCC1 and XPF in relation to overall survival of gastric cancer.

\begin{tabular}{|c|c|c|c|c|c|c|c|c|}
\hline \multirow[t]{2}{*}{ Gene } & \multicolumn{2}{|c|}{ Cases } & \multicolumn{3}{|c|}{ Events } & \multicolumn{3}{|c|}{ Overall Survival } \\
\hline & $\mathrm{N}=331$ & $\%$ & $\mathrm{~N}=103$ & & $\%$ & Median (month) & Log-rank & $\mathrm{HR}(95 \% \mathrm{CI})$ \\
\hline \multicolumn{9}{|c|}{ ERCC1 rs11615 } \\
\hline GG & 111 & 43.5 & 51 & 60 & 49.5 & 28.4 & & 1.0 (Reference) \\
\hline AG & 97 & 38.1 & 36 & 61 & 35 & 30.2 & & $0.69(0.38-1.26)$ \\
\hline AA & 47 & 18.4 & 16 & 31 & 15.5 & 33.2 & 0.16 & $0.60(0.28-1.30)$ \\
\hline \multicolumn{9}{|c|}{ ERCC1 rs2298881 } \\
\hline $\mathrm{AA}$ & 165 & 64.7 & 69 & 96 & 67 & 29.1 & & 1.0 (Reference) \\
\hline $\mathrm{AC}$ & 58 & 22.8 & 22 & 36 & 21.3 & 30.7 & & $0.85(0.44-1.64)$ \\
\hline $\mathrm{CC}$ & 32 & 12.5 & 12 & 20 & 11.7 & 31.5 & 0.25 & $0.83(0.35-1.93)$ \\
\hline \multicolumn{9}{|c|}{ XPF rs6498486 } \\
\hline AA & 174 & 68.2 & 79 & 95 & 76.7 & 26.4 & & 1.0 (Reference) \\
\hline $\mathrm{AC}$ & 55 & 21.6 & 20 & 35 & 19.5 & 31.6 & & $0.69(0.35-1.34)$ \\
\hline $\mathrm{CC}$ & 26 & 10.2 & 4 & 22 & 3.8 & 34.7 & 0.007 & $0.22(0.05-0.69)$ \\
\hline \multicolumn{9}{|c|}{ XPF rs2276465 } \\
\hline $\mathrm{CC}$ & 157 & 61.5 & 74 & 83 & 71.8 & 27.7 & & 1.0 (Reference) \\
\hline CG & 55 & 21.6 & 20 & 35 & 19.4 & 30.5 & & $0.64(0.32-1.26)$ \\
\hline GG & 43 & 16.9 & 9 & 34 & 8.8 & 33.6 & 0.01 & $0.30(0.12-0.69)$ \\
\hline
\end{tabular}

\section{DISCUSSION}

In this case-control study, we investigated whether the ERCC1 and XPF gene polymorphisms in the NER pathway were associated with the clinical outcome of gastric cancer treated with 5-Fu-based chemotherapy. We observed that the XPF rs6498486 CC genotype 
and the $X P F$ rs2276465 GG genotype were associated with a better response to chemotherapy and longer survival time in gastric cancer patients treated with 5-Fu-based chemotherapy. Our results demonstrated that the $X P F$ rs6498486 and $X P F$ rs2276465 polymorphisms can be used as predictive markers of response and expected to be valuable for individualizing patient treatment as this method enables discrimination of patients most likely to respond to combination therapy from those likely to be non-responsive.

ERCC1 is part of the NER complex involved in the repair of platinum/5-Fu-based induced interstrand and intrastrand cross-links (Gustavsson et al., 2009; Liang et al., 2010; Wang et al., 2012). Since ERCC1 acts on larger lesions covering 20-25 nucleotides, the efficient DNA repair capacity of ERCC1 appears to be a critical mechanism of the resistance to platinum/5-Fu-based drugs and radiation (Reed, 1998; Wood et al., 2001; Goode et al., 2002). Several previous studies indicated that the ERCC1 rs11615 polymorphism could be used as a prognostic factor in oxaliplatin-based treatment of this cancer (Matsubara et al., 2008; Wang et al., 2008; Ozkan et al., 2010; Liang et al., 2010; Yun et al., 2010; Yin et al., 2011). Two previous studies conducted in China indicated that the ERCC1 rs11615 polymorphism is associated with time to progression and OS of gastric cancer patients treated with oxaliplatin/5-Fu-based chemotherapy (Liang et al., 2010; Yin et al., 2011). However, the results are inconsistent. A recent study conducted in Korea indicated that ERCC1 protein expression does not appear to be associated with the prognosis of gastric cancer (Yun et al., 2010). Our study also indicated that there was no association between the ERCC1 rs11615 polymorphism and prognosis of gastric cancer.

$\mathrm{XPF}$, also known as ERCC4, is also involved in the NER pathway and is associated with susceptibility to xeroderma pigmentosum and a rare recessive syndrome that includes photosensitivity, malignant tumor development, and response to chemotherapy (Zhu et al., 2003). A previous study indicated that XPF is critical for recombination repair, mismatch repair, and possibly, immunoglobulin class switching, owing to its function in identifying damage sites (Kornguth et al., 2005). Therefore, polymorphisms of XPF may change the function of this gene and influence the response to chemotherapy. A previous study indicated that variations in $X P F$ were associated with treatment response in ovarian cancer, esophageal cancer, and gastric cancer (Fareed et al., 2010; DeLoia et al., 2012; Alexander et al., 2012). Only one study conducted in the United Kingdom had investigated the association between $X P F$ polymorphisms and the response to platinum-based chemotherapy (Fareed et al., 2010). However, this study did not identify the $X P F$ polymorphism as a predictor of response. In our study, we found that the $X P F$ rs6498486 CC genotype and the $X P F$ rs2276465 GG genotype were correlated with good response to chemotherapy. The inconsistency of these studies may be explained by differences in population backgrounds, and further studies with different populations are needed to confirm our findings.

This is the first study reporting an association between the polymorphisms $X P F$ rs6498486 and $X P F$ rs2276465 and response to oxaliplatin/5-Fu-based chemotherapy. ERCC1 rs11615 and $X P F$ rs2276465 may substantially contribute to the future design of individualized cancer treatment in gastric cancer patients. Therefore, further multicenter studies involving various populations are required to confirm our results.

\section{REFERENCES}

Alexander BM, Sprott K, Farrow DA, Wang X, et al. (2010). DNA repair protein biomarkers associated with time to 
recurrence in triple-negative breast cancer. Clin. Cancer Res. 16: 5796-5804.

Alexander BM, Wang XZ, Niemierko A, Weaver DT, et al. (2012). DNA repair biomarkers predict response to neoadjuvant chemoradiotherapy in esophageal cancer. Int. J. Radiat. Oncol. Biol. Phys. 83: 164-171.

Carles J, Monzo M, Amat M, Jansa S, et al. (2006). Single-nucleotide polymorphisms in base excision repair, nucleotide excision repair, and double strand break genes as markers for response to radiotherapy in patients with Stage I to II head-and-neck cancer. Int. J. Radiat. Oncol. Biol. Phys. 66: 1022-1030.

De Silva IU, McHugh PJ, Clingen PH and Hartley JA (2000). Defining the roles of nucleotide excision repair and recombination in the repair of DNA interstrand cross-links in mammalian cells. Mol. Cell Biol. 20: 7980-7990.

DeLoia JA, Bhagwat NR, Darcy KM, Strange M, et al. (2012). Comparison of ERCC1/XPF genetic variation, mRNA and protein levels in women with advanced stage ovarian cancer treated with intraperitoneal platinum. Gynecol. Oncol. 126: 448-454.

Fareed KR, Al-Attar A, Soomro IN, Kaye PV, et al. (2010). Tumour regression and ERCC1 nuclear protein expression predict clinical outcome in patients with gastro-oesophageal cancer treated with neoadjuvant chemotherapy. $\mathrm{Br}$. $J$. Cancer 102: 1600-1607.

Fleming ND, Agadjanian H, Nassanian H, Miller CW, et al. (2012). Xeroderma pigmentosum complementation group C single-nucleotide polymorphisms in the nucleotide excision repair pathway correlate with prolonged progressionfree survival in advanced ovarian cancer. Cancer 118: 689-697.

Friedberg EC (2001). How nucleotide excision repair protects against cancer. Nat. Rev. Cancer 1: 22-33.

Goode EL, Ulrich CM and Potter JD (2002). Polymorphisms in DNA repair genes and associations with cancer risk. Cancer Epidemiol. Biomarkers Prev. 11: 1513-1530.

Gustavsson B, Kaiser C, Carlsson G, Wettergren Y, et al. (2009). Molecular determinants of efficacy for 5-FU-based treatments in advanced colorectal cancer: mRNA expression for 18 chemotherapy-related genes. Int. J. Cancer 124: $1220-1226$.

International Agency for Research on Cancer (IARC) (2008). Globocan 2008: Stomach Cancer Incidence, Mortality and Prevalence Worldwide in 2008.

Kornguth DG, Garden AS, Zheng Y, Dahlstrom KR, et al. (2005). Gastrostomy in oropharyngeal cancer patients with ERCC4 (XPF) germline variants. Int. J. Radiat. Oncol. Biol. Phys. 62: 665-671.

Liang J, Li QF, Yao RY, Lu HY, et al. (2010). Association between genetic polymorphisms of ERCC1, XRCC1, GSTP1 and survival of advanced gastric cancer patients treated with oxaliplatin/5-Fu-based chemotherapy. Zhonghua Zhong Liu Za Zhi 32: 515-519.

MacDonald JS (2004). Clinical overview: adjuvant therapy of gastrointestinal cancer. Cancer Chemother. Pharmacol. 54 (Suppl 1): S4-11.

Matsubara J, Nishina T, Yamada Y, Moriwaki T, et al. (2008). Impacts of excision repair cross-complementing gene 1 (ERCC1), dihydropyrimidine dehydrogenase, and epidermal growth factor receptor on the outcomes of patients with advanced gastric cancer. Br. J. Cancer 98: 832-839.

Miller AB, Hoogstraten B, Staquet M and Winkler A (1981). Reporting results of cancer treatment. Cancer 47: 207-214.

Ozkan M, Akbudak IH, Deniz K, Dikilitas M, et al. (2010). Prognostic value of excision repair cross-complementing gene 1 expression for cisplatin-based chemotherapy in advanced gastric cancer. Asian Pac. J. Cancer Prev. 11: 181-185.

Reed E (1998). Platinum-DNA adduct, nucleotide excision repair and platinum based anti-cancer chemotherapy. Cancer Treat. Rev. 24: 331-344.

Vaezi A, Wang X, Buch S, Gooding W, et al. (2011). XPF expression correlates with clinical outcome in squamous cell carcinoma of the head and neck. Clin. Cancer Res. 17: 5513-5522.

Wang AT, Sengerova B, Cattell E, Inagawa T, et al. (2011). Human SNM1 A and XPF-ERCC1 collaborate to initiate DNA interstrand cross-link repair. Genes Dev. 25: 1859-1870.

Wang L, Wei J, Qian X, Yin H, et al. (2008). ERCC1 and BRCA1 mRNA expression levels in metastatic malignant effusions is associated with chemosensitivity to cisplatin and/or docetaxel. BMC Cancer 8: 97.

Wang Z, Chen JQ, Liu JL, Qin XG, et al. (2012). Polymorphisms in ERCC1, GSTs, TS and MTHFR predict clinical outcomes of gastric cancer patients treated with platinum/5-Fu-based chemotherapy: a systematic review. $B M C$ Gastroenterol. 12: 137.

Wohrer SS, Raderer M and Hejna M (2004). Palliative chemotherapy for advanced gastric cancer. Ann. Oncol. 15: 15851595.

Wood RD, Mitchell M, Sgouros J and Lindahl T (2001). Human DNA repair genes. Science 291: 1284-1289.

Yin M, Yan J, Martinez-Balibrea E, Graziano F, et al. (2011). ERCC1 and ERCC2 polymorphisms predict clinical outcomes of oxaliplatin-based chemotherapies in gastric and colorectal cancer: a systemic review and meta-analysis. Clin. Cancer Res. 17: 1632-1640.

Yun J, Kim KM, Kim ST, Kim JH, et al. (2010). Predictive value of the ERCC1 expression for treatment response and 
survival in advanced gastric cancer patients receiving cisplatin-based first-line chemotherapy. Cancer Res. Treat. 42: 101-106.

Zhu XD, Niedernhofer L, Kuster B, Mann M, et al. (2003). ERCC1/XPF removes the 3' overhang from uncapped telomeres and represses formation of telomeric DNA-containing double minute chromosomes. Mol. Cell 12: 1489-1498. 